\title{
PowerScribe 360 Mobile Radiologist App Review
}

\author{
Raja L. Gali ${ }^{1}$ Paras Lakhani ${ }^{2}$
}

Published online: 16 August 2016

(C) Society for Imaging Informatics in Medicine 2016

\section{App Specs}

App Icon URL: https://h3.googleusercontent.com/cKIf329 hqYRGBLNmp58-qzCJQqZ_p-knZPD4uJ8ESPzLmeXx0 adn_EGGWEXJ0gAWAJs=w300-rw

App name: Powerscribe 360 Radiologist

App developer: Nuance Communication, Inc

App developer website: http://www.nuance.com/ products/powerscribe360/index.htm

App price: Free

Apple App Store URL: https://itunes.apple.com/us/ app/powerscribe-360-radiologist/id520847596? $\mathrm{mt}=8$

Google Play Store URL: https://play.google.com/ store/apps/details?id=com.nuance.radiologist\&hl=en

Category: Medical

Tags: \#medical \#radiology

Works offline: $\mathrm{N}$

FDA approval: N/A

Promotion code: None

\section{Quick Review}

(1 star: lowest / 5 stars: highest)

Overall rating (1-5): 4

Raja L. Gali

Raja.Gali@Jefferson.edu

Paras Lakhani

Paras.Lakhani@Jefferson.edu

1132 South 10th Street, Suite 877GC, Philadelphia, PA 19107, USA

2132 South 10th Street, Suite 796C, Philadelphia, PA 19107, USA
Content (1-5): 4

Usability (1-5): 4

Pros: simple and user-friendly interface, easy to read fonts, secure access to diagnostic reports to edit and finalize, and access to radiology-specific reference materials via Assisted Diagnosis, speech-to-text functionality works good.

Cons: long sign-on time, takes a long time to open a report for editing, cannot forward or assign to another radiologist, and cannot see history of signed reports, no notification alerts, unable to create a new report.

At a glance: Secure app that gives easy access to reports for editing and signoff using a smartphone or a tablet. User can build a work list based on different criteria. The app also gives easy access to radiology-specific reference materials through Assisted Diagnosis.

\section{Full Review}

\section{Introduction}

Powerscribe 360 Radiologist by Nuance Communications, Inc., is an app that is available for download on both iOS and Android devices, including phones and tablets. The app is designed for authorized users to edit, addend, or sign off an existing report.

\section{Purpose/Features/Content}

The mobile app allows the radiologist to view a pre-existing report that has already been started on a PC by the radiologist themselves or when a report has been assigned to them by a resident or fellow. These reports appear in their "Sign Queue" (Fig. 1). 


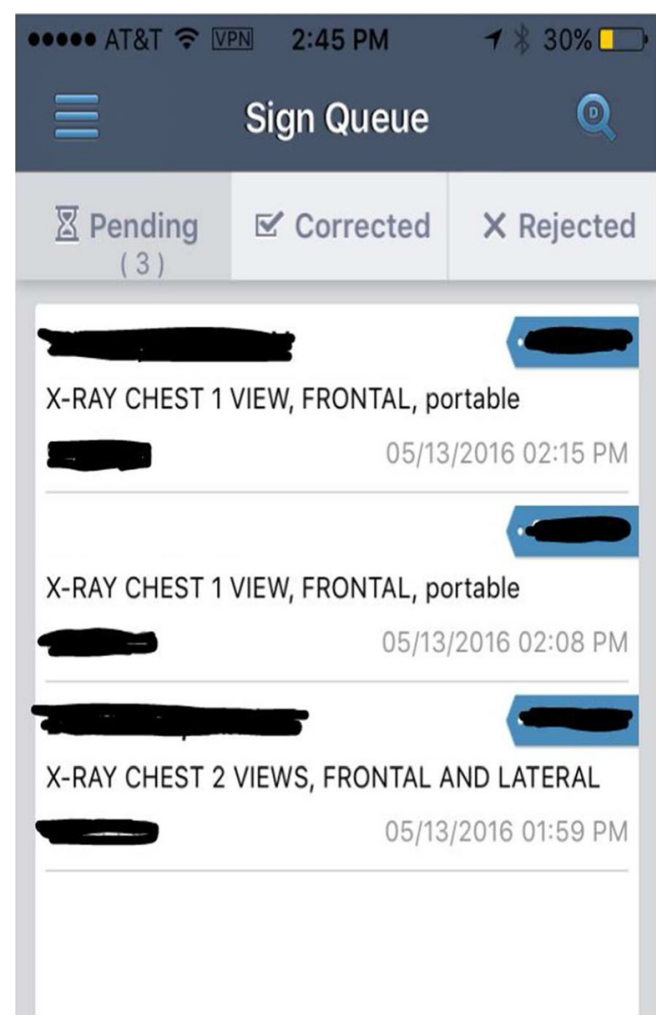

Fig. 1 Sign queue

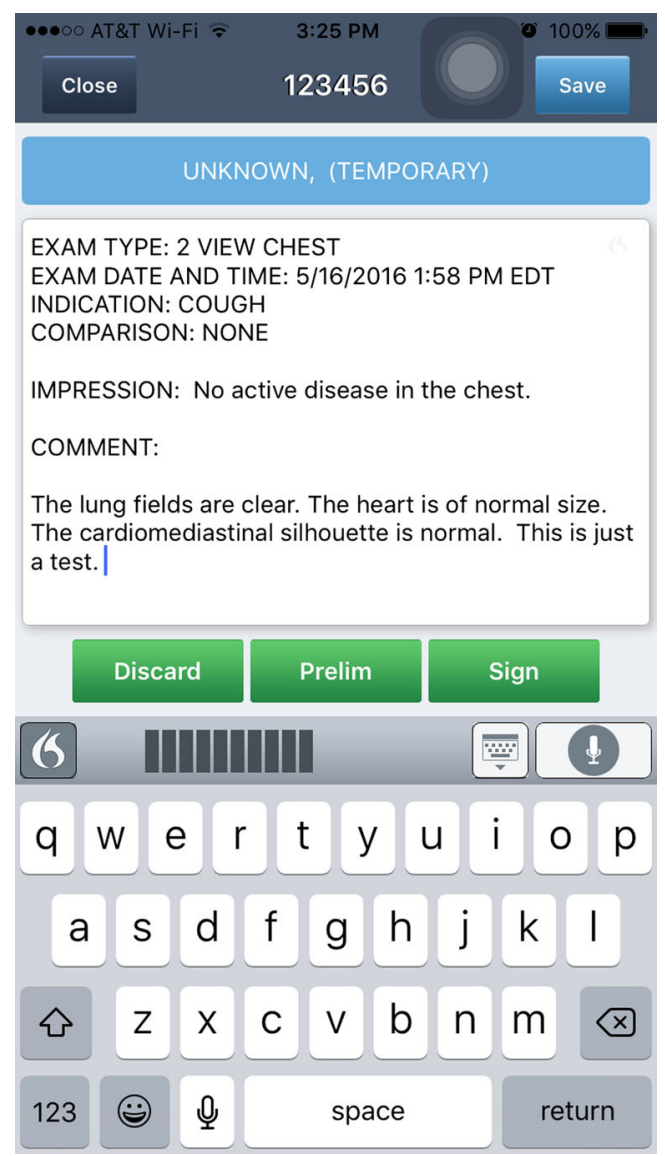

Fig. 2 Keyboard
Edits to the reports can be made using the keypad functionality of the mobile device (Fig. 2). The application also has full speech-to-text functionality (Fig. 3), which was tested extensively on an iPhone 5 using iOs 9.3.2 over Wi-Fi and was demonstrated to perform comparably to the PC-based application. This testing included ten $\mathrm{CT}$ reports, which contained many medical terms often used in radiology, such as "Fleischner Society Guidelines," "bronchiectasis," and "atherosclerotic calcifications," which were transcribed with near- $100 \%$ accuracy. There is a brief delay in transcribing the dictated words, but this is no more than $1 \mathrm{~s}$. The application was also noted to contain many of the features present in the Windows-based program, such as voice-enabled commands such as "scratch that," and automatic date/time. PS360 app sends the audio recordings from the mobile device to its secure cloud server, which translates it to text. This performed fairly well over our hospital's Wi-Fi.

The speech-to-text function was tested in both a relatively noisy environment with lots of background noise and in a quiet environment, and performed equally well. Measurement data also transcribed accurately including numerical, "cm" and "mm" unit designations.

The server is also updated relatively quickly while using the Mobile Radiologist app. Saving an edited report on the

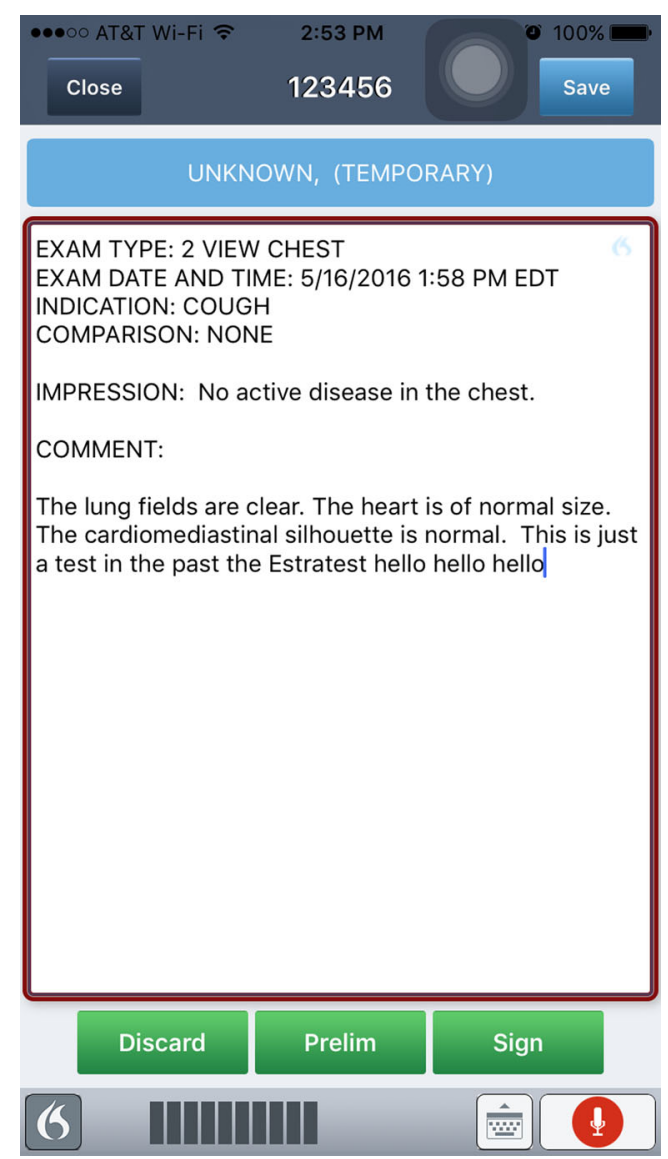

Fig. 3 Microphone 
mobile device using the app reflected a near-immediate change in the Windows-based application, under $2 \mathrm{~s}$. Changes made to a report and saved on Windows-based application also got reflected on the app relatively quickly. Also, users can be logged into the Windows-based application and the mobile app at the same time.

Users can build work lists based on ordering physician, modality, patient type, and time frame. The pending tab lists all the reports that have been assigned to the attending radiologist.

Opening a report for editing takes about $7 \mathrm{~s}$ and signing off takes about $3 \mathrm{~s}$. When a report is opened for editing, radiologist can listen to the initial voice dictation (Fig. 4). After editing the report, radiologist can sign the report as final or as a prelim. The radiologist can discard the report on the app (Fig. 5).

The app also gives access to radiology-specific reference materials through Assisted Diagnosis with links to medical content sources. Assisted Diagnosis basically enters the search term (e.g., lipoma) into one of the following six search engines:
1. Google (worked)
2. Google Books (worked)
3. MedPix (did not work)
4. CHORUS (did not work)
5. YottaLook (worked)
6. Aunt Minnie (worked)

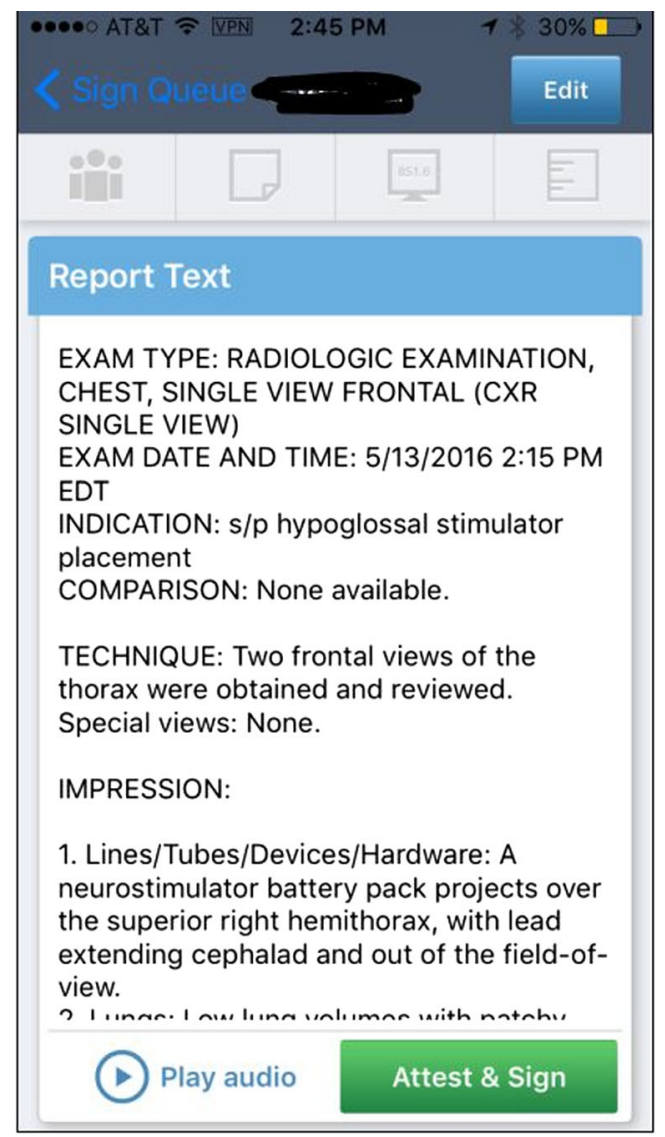

Fig. 4 Play audio

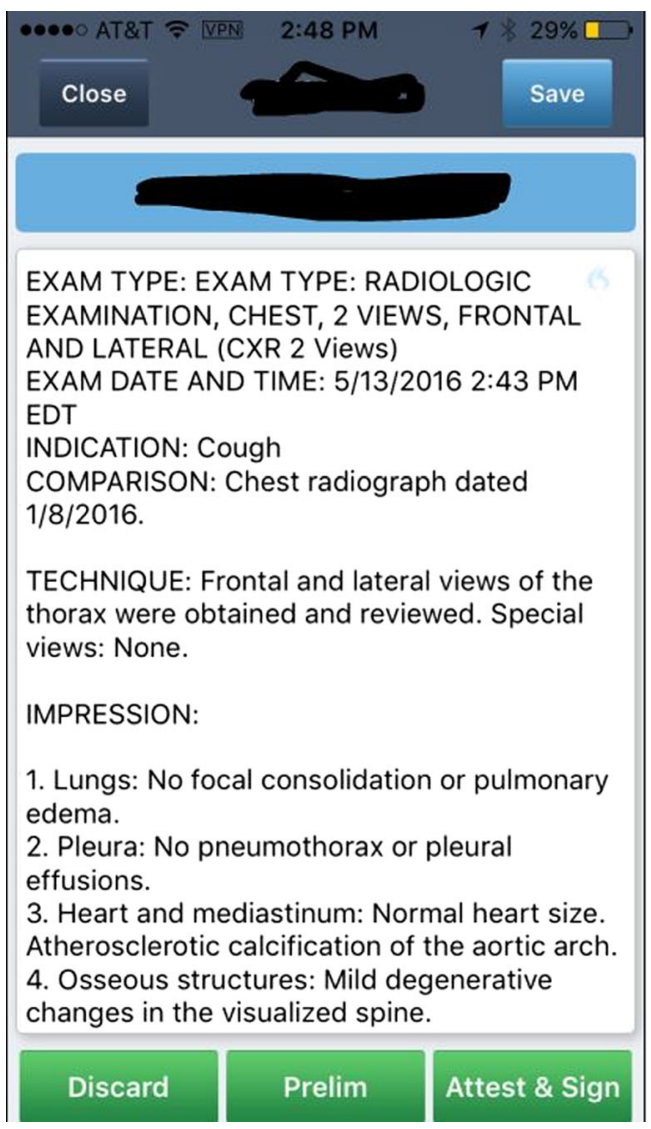

Fig. 5 Discard/sign/prelim

Assisted Diagnosis was functional but there was nothing special about it other than passing the search terms to the respective search engines.

\section{Usability}

The app offers an intuitive and easy-to-use interface. Editing uses the on-screen keyboard or voice dictation using the inbuilt microphone of the smartphone or tablet. Speech-totype feature works good both in noisy and silent environments. Radiologist can edit, finalize, and addend a report.

\section{Good}

Intuitive user interface with basic, easy to navigate menu, speech works great, time-saver for signing preliminary reports.

Neutral: Assisted Diagnosis provides useful links to different radiology-specific content and provides simultaneous results.

\section{Room for Improvement}

While the app provides an easy and secure access to reports, the login time is long. The initial login to the application takes 
about $30 \mathrm{~s}$. Since the app logs the users out every time the app is not being actively used, this long login time can be frustrating. The user is logged out even if a notification pops up on the screen while the app is being used actively; edits that were made on the draft are not saved when the user is logged out. The user is also unable to see a history of signed cases, forward cases to another user, generate new reports, and receive notification alerts, such as when a preliminary report is available to sign. 